\title{
Realization Feature of Mesenchymal Dermal Cells Tissue Engineering Construction Response in Granulating Wound Transplantation in Relation with Time-Frame
}

\author{
Elena Petersen ${ }^{1,2}$ \\ ${ }^{1}$ Moscow Scientific Research Center of Dermatovenerology and Cosmetology of Moscow City, Moscow, Russia; ${ }^{2}$ Institute of Gen- \\ eral Pathology and Pathophysiology RAMS, Moscow, Russia. \\ Email: petersen.elena.v@gmail.com
}

Received June $8^{\text {th }}, 2012$; revised July $11^{\text {th }}, 2012$; accepted July $25^{\text {th }}, 2012$

\begin{abstract}
Derma is progenitor cells sours, which are able to differentiate further in several mesodermal lineage and neural and endodermal lineage. Culture conditions, skin taking site and culture medium composition considerably contribute to it. Spheroid cultured mesenchymal dermal cells contribution to skin regeneration in granulating wound in rat model was estimated.
\end{abstract}

Keywords: Brief Report; 3D Cultivated Tissue Engineering Construction; Skin; Mesenchymal Dermal Cells; Sprouting Capillary-Like Structures

\section{Introduction}

Derma is progenitor cells sours, that are able to differentiate further in several mesodermal lineage (osteogenic, chondrogenic) [1-4] and neural and endodermal lineage (endotheliocyte, hepatocyte, islet cell) [5,6]. Culture conditions, skin taking site and culture medium composition considerably contribute to it. Spheroid cultured mesenchymal dermal cells contribution to skin regeneration in granulating wound in rat model was estimated.

\section{Methods}

\subsection{Cellular Construction}

3rd - 4th passage mesenchymal dermal cells were untainted in accordance with standard protocol after plastic surgical operations on donors given the informed concerned. Hanging drop method was used for spheroid cells incubation. $25 \mu \mathrm{l}$ (for $90 \mathrm{ml}$ plates) (Corning) of suspendsion and 5 - $6 \mu$ l Dulbecco's Modified Eagle Medium $(\mathrm{DMEM}) / \mathrm{F} 12$ (Sigma) with antibiotic Invitrogen were added to incubate hanging drops in standard conditions $\left(37^{\circ} \mathrm{C}, 5 \% \mathrm{CO}_{2}\right)$ for 7 days. Preliminarily mesenchymal cells monolayer staining in recommended protocol was used for transplanted cells visualization. Scores dilution considered as minimal and not taken in account because of Spheroid type cells resting stage reaching and short checking point time-frame.

\subsection{Animal Model}

Wistar rat were used in experiment with human treatment rule observance. Wound surface was formed with fullthickness round incision up to fascia of $5 \mathrm{~mm}$ in back area. Round rings with boarding edge were sewed against contraction wound healing. Spheroid cells were transplanted after wound formation. on the 4th day Rings were removed for wound epithelization. The evaluation on the 4 th, 10th days was based on the ground of morphometry, immunohistochemistry data, epithelization area and rate in comparison with intact control. Standard paraffin sections histological study was used. Immunohistochemistry study was made with primary antibodies to angiogenic factors, to CD31, VEGF (Dako), Flk-1 (Chemicon) and fluorescein isothiocyanate conjugated secondary antibodies, FITC, (Alexa Fluor ${ }^{\circledR}$ 488). For nucleus visualization Hoechst 33,342 (Sigma) dye was used. In immunohistochemistry, cluster of differentiation 31 (CD31) also known as platelet endothelial cell adhesion molecule (PECAM-1) is used primarily to demonstrate the presence of endothelial cells in histological tissue sections. Vascular endothelial growth factor (VEGF) is a signal protein produced by cells that stimulates vasculogenesis and angiogenesis and, together a receptor for vascular endothelial growth factor (Flk-1) is a marker of endothelial cells.

\section{Results}

Preferential spheroid disposition was registered in upper 
and middle granulation tissue layers in vessel walls area (Figure 1). Some cells remained spheroid while others migrated to matrix. Migrated cells response was found different by 4 th day. Sprouting capillary-like structures were detected in defect center where less fibrillar extracellular matrix was generated (Figure 2). Distant from granulation wound center Spheroids in deeper layers diffusely disseminated (Figure 3). Transplanted mesenchymal dermal cells formed long bands and tubular form structures by 10th day. Spheroids remaining their form were found in some areas of wound. Small part of them moved to surrounding matrix.

Immunohistochemistry study shows angiogenic markers expression (CD31, Flk-1, VEGF) in tubular structure forming and spheroid form remaining and diffusion cases (Figure 4).

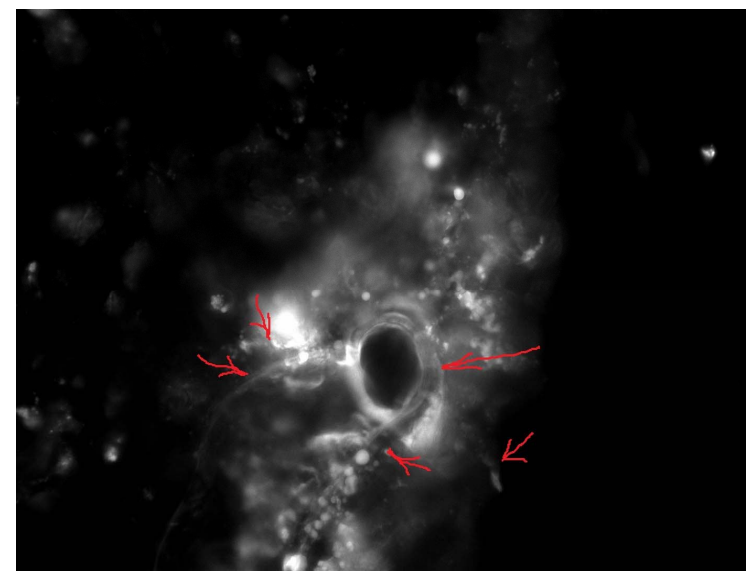

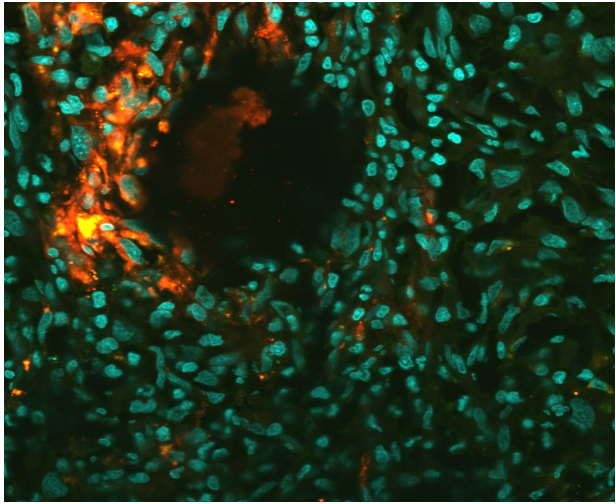

Figure 1. Mesenchymal dermal cells, fluorescent membrane tracer Dil (red glow) marked, cell nuclei are marked blueHoechst 33,342 stain. 4 days after transplantation. Vessel walls area cell disposition.

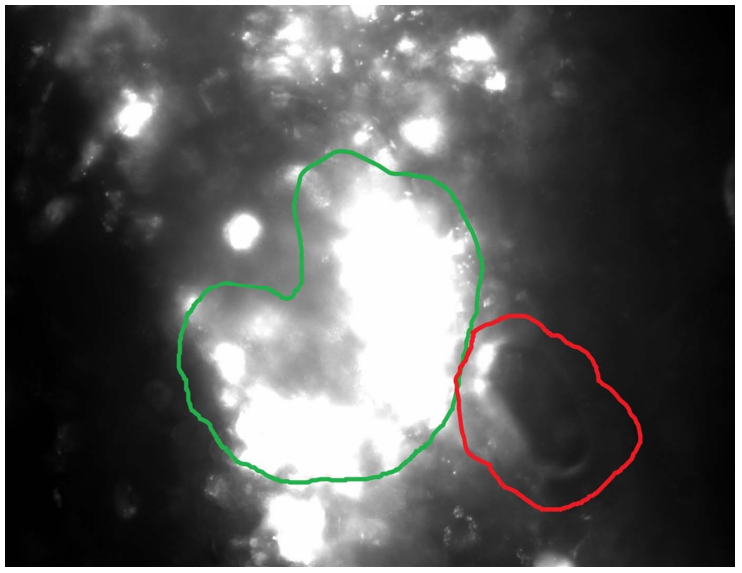

Figure 2. Granulation wound upper central part criosection with transplanted mesenchymal dermal cells marked with fluorescent membrane tracer 4th day after transplantation. Pictures made with monochrome matrix for better contrast. White parts match fluorescent transplanted cells, spheroid organized cells have intense glow, cells migrated to matrix or cell longitudinal extension and cell proliferation have less glow. Sprouting capillary-like structures are marked with red arrows. Stage of Spheroid rearrangement and migration is marked green. Sprouting capillary-like structures forming cells are marked red.
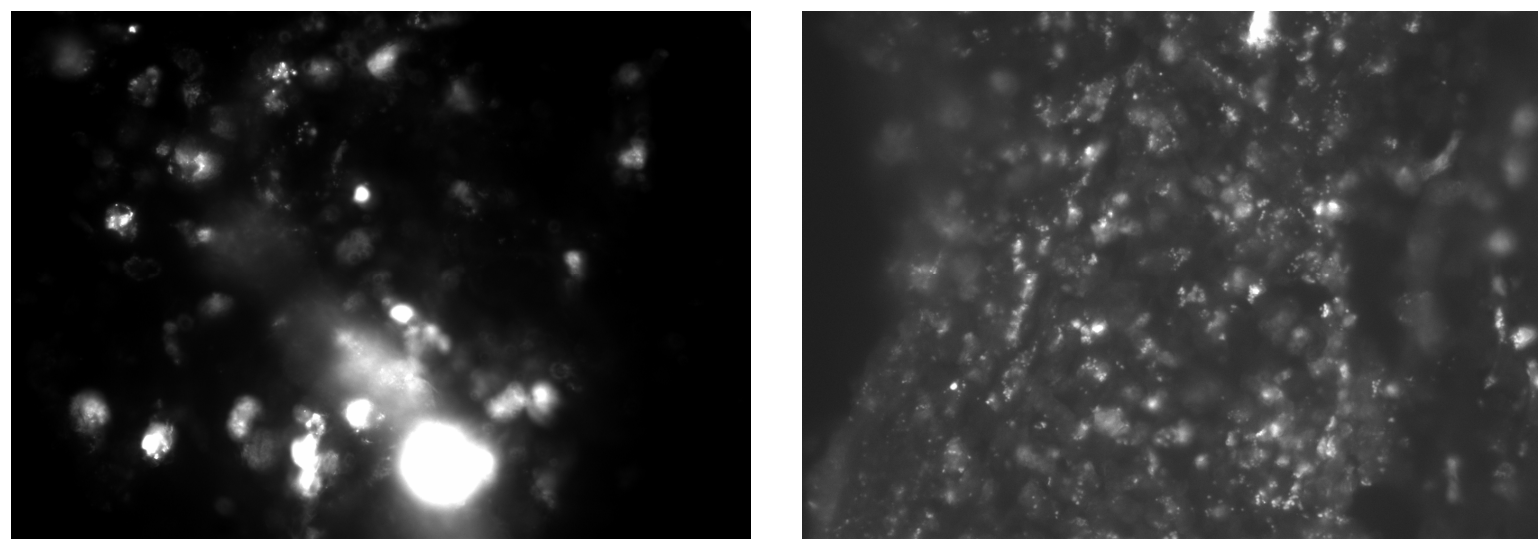

Figure 3. Granulation wound middle peripheral part criosection with transplanted mesenchymal dermal cells marked with fluorescent membrane tracer 4th day after transplantation. Pictures made with monochrome matrix for better contrast. White parts match fluorescent transplanted cells, spheroid organized cells have intense glow, cells migrated to matrix or cell longitudinal extension and cell proliferation have less glow. Tubular form structure goes diagonally from spheroid placed in lower part. Diffuse migrated cell are clearly visible round and right. 

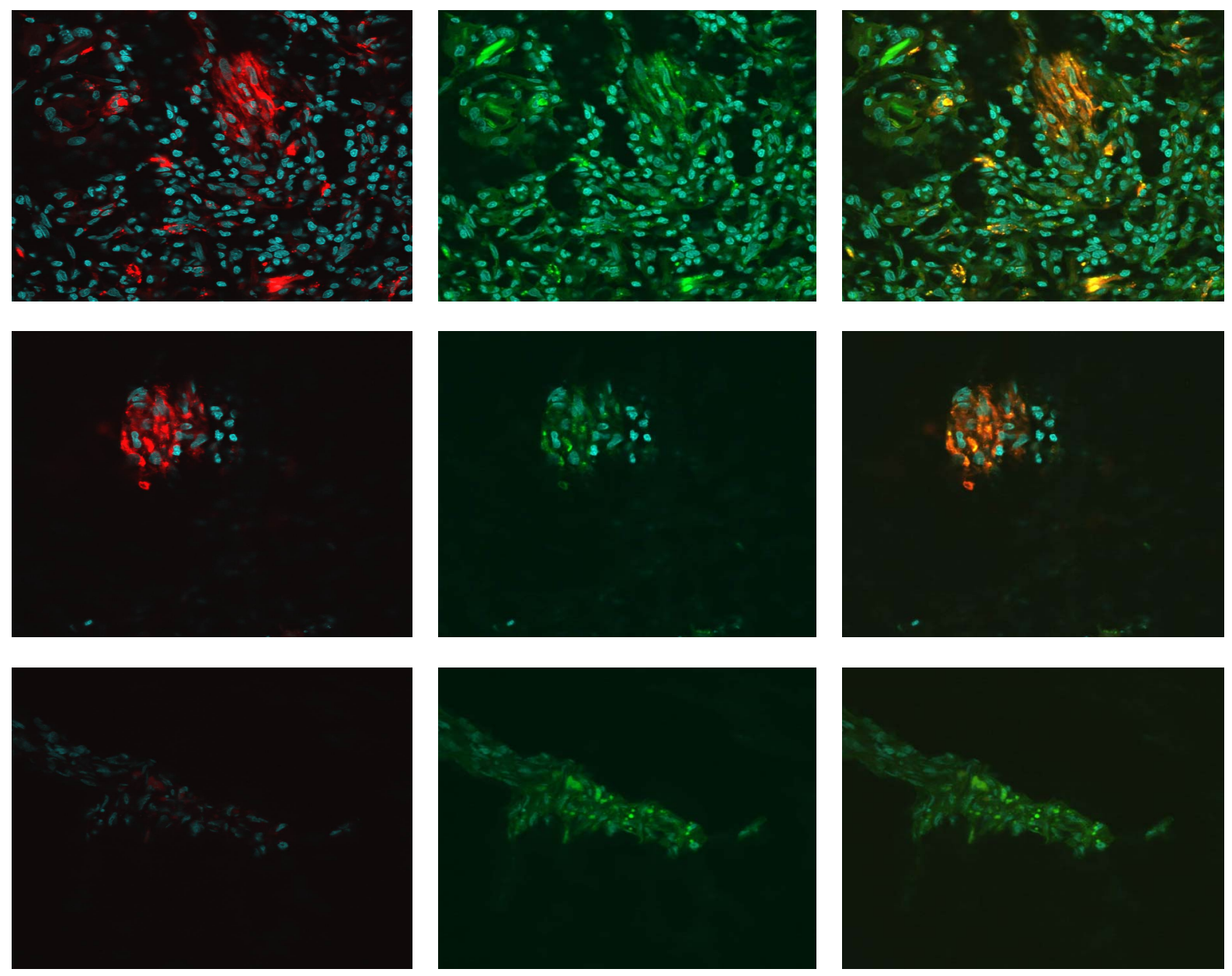

Figure 4. Immunohistochemical staining for VEGF. Cells marked with lipotracer Dil have red glow, VEGF expressing cellsgreen glow, combined channels are right positioned. Cell nuclei are marked blue-Hoechst 33,342 stain. Upper line- diffuse cells, middle line—spheroid cell, lower line-tubular form structures (confocal microscopy).

\section{Discussion}

3D spheroid mesenchymal dermal cells actualize ephithilial—mesenhimal transition [7]. Upper spheroid layers playing defense role boarder spheroid tissue microenvironment. These cell type migrates to surrounding matrix first. Endothelial or epithelial predifferentiation of upper layer cell can explain sprouting capillary-like structures forming on first stages of spheroid dissemination.

Spheroids cultivated in same conditions in vitro divide in two-fast and slow matrix disseminating types. First rapidly reflects to changed conditions in wound and matrix induction signals. At first phase wound healing extracellular matrix lacks ordered fibrous proteins. That fact and soluble signal factors during first phase traumatic process cause endothelial differentiation of superficial migrated cell. Early in vitro experiments we showed that VEGF-induced spheroids derived from the stromal cells of the skin dermis, placed in Matrigel did not form sprouting capillary-like structures, although it is known that the induction of VEGF spheroids derived from umbilical cord stromal cells leads to the appearance of sprouting capillary-like structures structures in Matrigel [8]. Second spheroid type slowly reacts to surrounding changes and cells migrate later. Matrix has another composition when cell in these spheroids are activated. As a result migrated cell make fibroblast form phenotype and build into matrix diffusely.

\section{Conclusion}

Studying 3D cultivated tissue engineering construction helps to understand reparative regeneration processes and create manageable induction technologies.

\section{REFERENCES}

[1] J. Toma, M. Akhavan, K. Fernandes, et al., "Isolation of Multipotent Adult Stem Cells from the Dermis of Mammalian Skin," Nature Cell Biology, Vol. 3, No. 9, 2001, pp. 778-784. 
[2] J. Toma, I. McKenzie, D. Bagli, et al., "Isolation and Characterization of Multipotent Skin-Derived Precursors from Human Skin," Stem Cells, Vol. 23, No. 6, 2005, pp. 727-737. doi:10.1634/stemcells.2004-0134

[3] J.-F. Lavoie, J. Biernaskie, Y. Chen, et al., "Skin-Derived Precursors Differentiate into Skeletogenic Cell Types and Contribute to Bone Repair," Stem Cells and Development, Vol. 18, No. 6, 2009, pp. 893-905. doi: $10.1089 / \mathrm{scd} .2008 .0260$

[4] M. Sieber-Blum, M. Grim, Y. Hu and V. Szeder, "Pluripotent Neural Crest Stem Cells in the Adult Hair Follicle," Developmental Dynamics, Vol. 231, No. 2, 2004, pp. 258-269. doi:10.1002/dvdy.20129

[5] F. Chen, W. Zhang, D. Bi, et al., "Clonal Analysis of Nestin $^{-}$Vimentin ${ }^{+}$Multipotent Fibroblasts Isolated from
Human Dermis," Journal of Cell Science, Vol. 120, 2007, pp. 2875-2883. doi: $10.1242 /$ jcs. 03478

[6] D. Bi, F. Chen, W. Zhang, D. Bi, et al., "Differentiation of Human Multipotent Dermal Fibroblast into Islet-Like Cell Cluster," BMC Cell Biology, Vol. 11, No. 46, 2010, pp. 1-7.

[7] I. Saburina and V. Repin, "3D-Culturing from Single Cells to the Regeneration of Tissue (the Question about the Phenomenon of Epithelial-Mesenchymal Plasticity)," Cell Transplantation and Tissue Engineering, Vol. 5, No. 2, 2010, pp. 75-86.

[8] A. Gorkun, I. Saburina, N. Kosheleva, A. Pulin and V. Repin, "Induction of Vasculogenesis in the Spheroid of the Stromal Cells of Umbilical Cord," Tula Journal New Medical Technologies, 2012, in press. 